\title{
Cast Bronchitis in a Cystic Fibrosis Child: Experience with Direct Instillation of Dornase Alfa as an Adjunct Treatment Agent
}

\author{
Sedigheh Yousefzadegan ${ }^{1}$, Rohollah Shirzadi $\mathbb{B}^{1,2}$, Safura Navaie ${ }^{1,2}$, Alireza Takzare ${ }^{3}$, Mahdieh \\ Ghaempanah ${ }^{1}$ and Mohammadreza Modaresi (iD) ${ }^{1,2, *}$ \\ ${ }^{1}$ Pediatric Pulmonary and Sleep Medicine Department, Children's Medical Center, Tehran University of Medical Sciences, Tehran, Iran \\ ${ }^{2}$ Department of Pediatrics, Tehran University of Medical Sciences, Tehran, Iran \\ ${ }^{3}$ Pediatric Anesthesiology Department, Children's Medical Center, Tehran University of Medical Sciences, Tehran, Iran \\ "Corresponding author: Pediatric Pulmonary and Sleep Medicine Department, Children's Medical Center, Tehran University of Medical Sciences, Tehran, Iran. Tel: \\ +98-9131134710, Email: mr-modaresi@sina.tums.ac.ir
}

Received 2019 January 21; Revised 2019 June 10; Accepted 2019 June 28.

\begin{abstract}
Introduction: Lobar atelectasis is one of the common problems in patients with cystic fibrosis and can be a cause of respiratory symptoms in these patients even in the absence of bacterial infection.

Case Presentation: Here we introduce a 9-year-old boy with cystic fibrosis who had many admissions because of respiratory distress due to lung collapse mainly caused by plastic bronchitis.

Conclusions: In a number of patients with cystic fibrosis, lobar atelectasis is seen which is resistant to medical treatments. Early fiberoptic bronchoscopy as a measure for mechanical removal of the blocking agent and sometimes direct injection of drugs like dornase alfa into the bronchus is a successful modality in the treatment of lobar collapses in chronic pulmonary diseases.
\end{abstract}

Keywords: Cystic Fibrosis, Atelectasis, Plastic Bronchitis, Fiberoptic Bronchoscopy, Dornase Alfa, N-Acetyl Cysteine

\section{Introduction}

Lobar atelectasis is one of the common problems in patients with cystic fibrosis and can be a cause of respiratory symptoms in these patients even in the absence of bacterial infection $(1,2)$. Bronchial plaque mucus, also referred to as plastic bronchitis or cast bronchitis, is a rare but threatening condition, and in many cases requires aggressive and rapid treatment (1-5).

\section{Case Presentation}

A 9-year-old boy was diagnosed with pneumonia and hospitalized in Shiraz since 6 months of age, with two measures of sweat chlorine more than 100 . He was being monitored with cystic fibrosis diagnosis since then. According to parents, he did not have any specific pulmonary problem until he was 7 years old and until his hospitalization he has never had any sputum culture or screening. At 7, he was admitted to a clinic in Ahwaz with coughing and respiratory distress. Following the exacerbation of symptoms and occurrence of shortness of breath, chest X-ray showed a complete collapse of the left lung (Figure 1A and B), so the patient was sent to Tehran for treatment.
In fiberoptic bronchoscopy (FOB) the airways contained thick mucus and abundant plaques. After washing and evacuation, collapse of the lung was resolved. At that time, CT scan showed no evidence of bronchiectasis. The patient's sputum culture was not positive but according to his symptoms the patient received venous empiric treatment with antibiotics for 2 weeks and was discharged in good general condition. From that time on, he received two hyper saline nebulizers daily and chest physical therapy. Despite the aforementioned measures, one year later, he again suffered from respiratory symptoms and collapse of the upper lobe of right lung that was not responsive to lung physiotherapy and had to be treated with lung washing. Negative sputum culture was reported. The patient has since been treated with dornase alfa nebulization in addition to previous treatments.

Ten months later, the patient gradually lost lung function and his shortness of breath returned. In the imaging, the collapse of the right upper lobe and the lower lobe of the left lung were seen.

Even intensive physiotherapy was ineffective. According to the previous history and relative symptoms, the patient underwent an early fiberoptic bronchoscopy at 

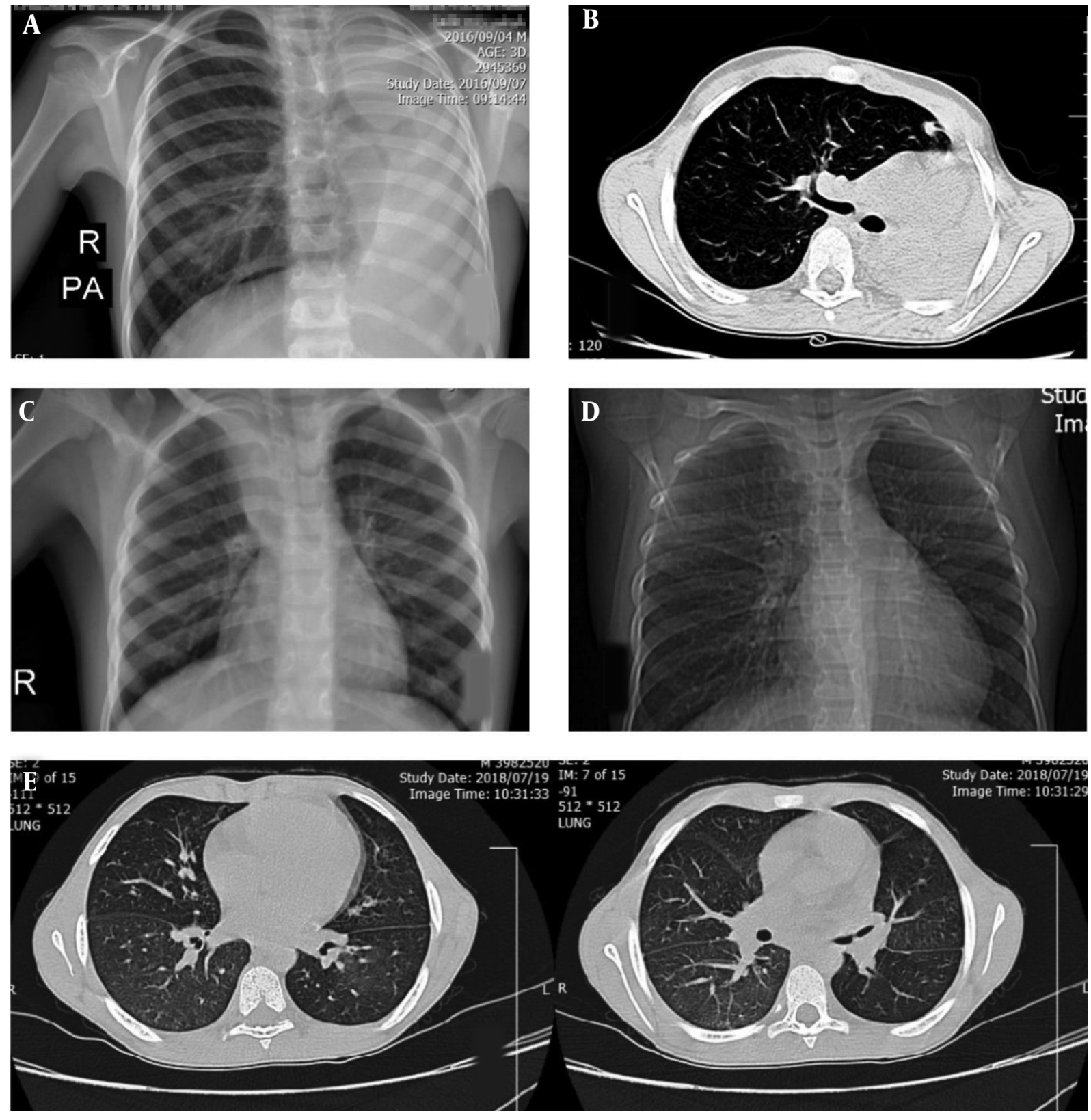

Figure 1. A and B, full lung collapse followed by obstruction with plaque in CXR and CT scan; C, partial opening of whole lung collapse with FOB and NAC injection; D, collapse removal after dornase alpha injection in the site; E: the early stages of lung bronchiectasis.

the Children's Medical Center Hospital. Mucus plaque appeared in the form of a cyst in the airways, which could not be removed by normal saline washing. Injection of $\mathrm{N}$ acetyl cysteine (diluted with normal saline by 1:2 ratio) was performed. A large number of mucus plaques (cast bronchitis) was removed (Figure 2) and the patient was placed under lung physical therapy. In spite of these measures, in the control radiography, the collapse of the upper lobe of right lung and the middle segment of the lower left lobe were still visible (Figure $1 \mathrm{C}$ ), so the patient became a candidate for FOB again.

During the fiberoptic bronchoscopy, redistribution of abundant and concentrated secretions was observed, which was, of course, easier to be suctioned after the first wash. In the upper lobe of the right lung a mucus cast was seen, which despite frequent attempts of washing with 

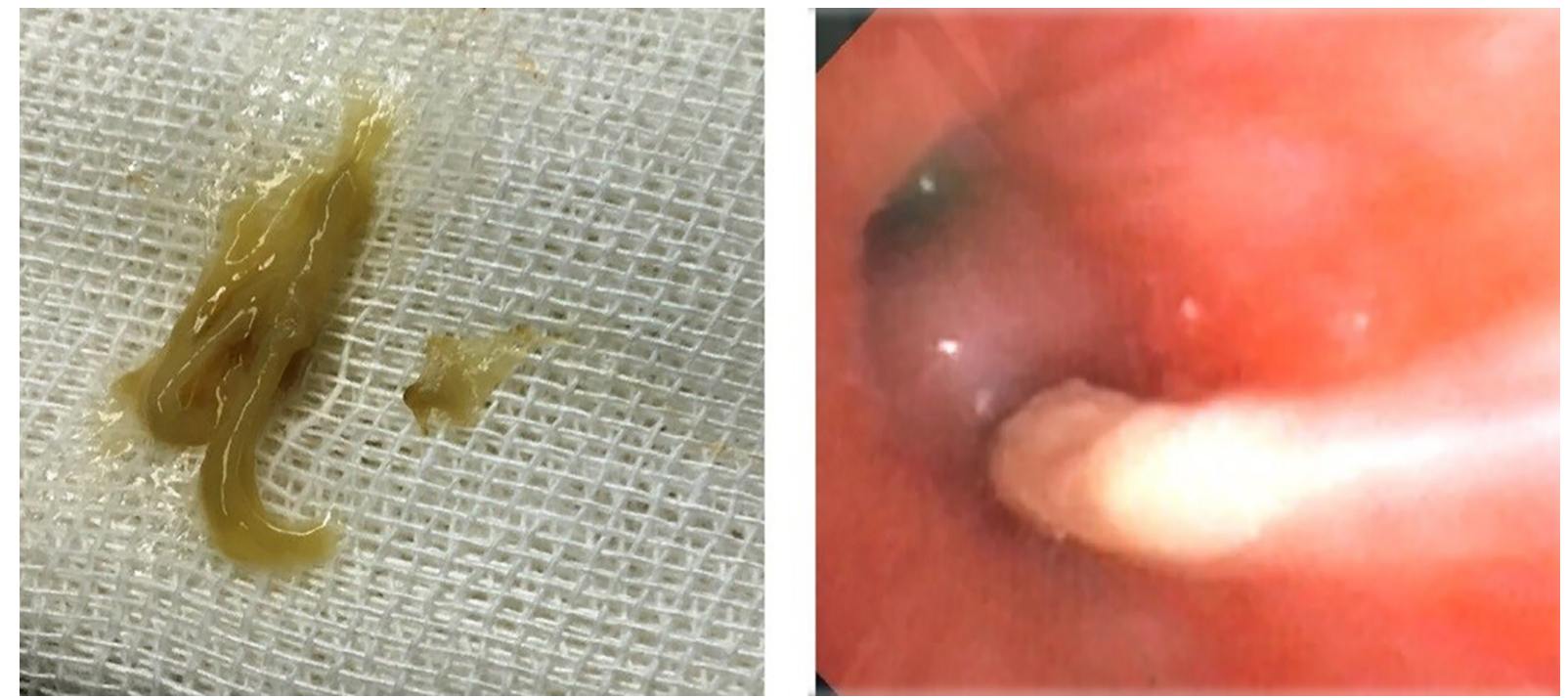

Figure 2. Left, cast bronchitis; right, mucus loosed after instillation of dornase alfa

normal saline and n-acetyl cysteine could not be removed. This time, direct injecting of dornase alfa took place. After a few minutes, the plaque began to gently slip off (Figure 2) and was completely removed by re-washing with normal saline (Figure 1D). In a study with pulmonary CT scan, increased peribronchial thickening and progression to the early stages of bronchiectasis was seen which could be due to prolonged bronchial obstruction (Figure 1E). Sputum and bronchial secretion cultures were negative. Eventually the patient was discharged in a good general condition.

An informed consent was obtained for any experimentation with this patient and all experiments were performed in compliance with institutional guidelines.

\section{Discussion}

In a number of patients with cystic fibrosis, lobar atelectasis is seen which is resistant to medical treatments. Although the precise cause of mucosal placement of casts such as "plastic bronchitis" is still unknown $(3,5)$, early fiberoptic bronchoscopy as a measure for mechanical removal of the blocking agent is very helpful in these cases, leading to decrease in hospital stay and improving the quality of life of patients $(1,4,6)$. Now, direct injection of the drug into the bronchus is an important and successful modality in the treatment of lobar collapses in chronic pulmonary diseases. In this regard, the use of n-acetyl cysteine and dornase alfa $(2,5-7)$, can be mentioned which several patients, including the patient above, have been treated with this method in this center.

\section{Footnotes}

Conflict of Interests: The authors declare no conflict of interests.

Funding/Support: There was no funding for this study. Patient Consent: The patient's consent had been taken.

\section{References}

1. Madappa T, Kamangar N. Atelectasis treatment \& management. MedScape; 2018. Available from: https://emedicine.medscape.com/ article/296468-treatment.

2. McLaughlin AM, McGrath E, Barry R, Egan JJ, Gallagher CG. Treatment of lobar atelectasis with bronchoscopically administered recombinant human deoxyribonuclease in cystic fibrosis? Clin Respir J. 2008;2(2):123-6. doi: 10.1111/j.1752-699X.2007.00024.x. [PubMed: 20298317].

3. Salamone I, Mondello B, Lucanto MC, Cristadoro S, Lombardo M, Barone M. Bronchial tree-shaped mucous plug in cystic fibrosis: Imaging-guided management. Respirol Case Rep. 2017;5(2). e00214. doi: 10.1002/rcr2.214. [PubMed: 28096997]. [PubMed Central: PMC5233562].

4. Studer SM, Terry PB. Images in clinical medicine. Bronchial cast. $N$ Engl J Med. 2002;346(13):981. doi: 10.1056/NEJMicm010283. [PubMed: 11919307].

5. Mateos-Corral D, Cutz E, Solomon M, Ratjen F. Plastic bronchitis as an unusual cause of mucus plugging in cystic fibrosis. Pediatr Pulmonol. 2009;44(9):939-40. doi: 10.1002/ppul.21063. [PubMed: 19658112].

6. Kumar A, Jat KR, Srinivas M, Lodha R. Nebulized N-acetylcysteine for management of plastic bronchitis. Indian Pediatr. 2018;55(8):701-3. doi: 10.1007/s13312-018-1363-8. [PubMed: 30218522].

7. Shah PL, Scott SF, Hodson ME. Lobar atelectasis in cystic fibrosis and treatment with recombinant human DNase I. Respir Med. 1994;88(4):313-5. doi: 10.1016/0954-6111(94)90063-9. [PubMed: 8036296] 